\title{
A Review on Drugs which Induce Liver Disease
}

\author{
Kumar A, Ashwlayan VD* and Verma M \\ Department of Pharmaceutical Technology, Meerut Institute of Engineering and \\ Technology, Dr. APJ Abdul Kalam Technical University, India
}

*Corresponding author: Dr. Vrish Dhwaj Ashwlayan, Department of Pharmaceutical Technology, Meerut Institute of Engineering and Technology NH-58, Meerut, Uttar Pradesh, India, Tel: 91-9412493228; Email: vrish.ashwlayan@miet.ac.in

\section{Review Article}

Volume 3 Issue 2

Received Date: July 13, 2018

Published Date: July 23, 2018

\section{Abstract}

The number of drugs associated with adverse reactions involving the liver is extensive. One of the more common reasons for the withdrawal of a drug from the marketplace is an elevation of liver enzymes. Alcohol-induced liver disease is the most common type of drug-induced liver disease. All other drugs together account for less than $10 \%$ of patients hospitalized for elevated liver enzymes. In approximately $75 \%$ of these cases liver transplantation is ultimately required for patient survival. The liver's function affects almost every other organ system in the body. It is important to know the patterns of drug-related pathology in order to assess adverse reactions when they occur. It is also important to understand how and when to monitor for these reactions. For some drugs, a genetic or acquired abnormality must exist in a particular metabolic pathway for a toxic reaction to take place in other cases, the reactions are typically associated with a drug concentration and often respond to simply lowering the dose of the drug. Idiosyncratic reactions tend to occur without association to particular blood concentrations or specifically identified metabolic abnormalities. For example, sulfonylureas like glipizide and antibiotics like ciprofloxacin have caused severe liver disease, resulting in the need for transplantation in a very small group of patients. Idiosyncratic reactions are rare and are sometimes described as liver hypersensitivity to a drug.

Keywords: Hypersensitivity; Sulfonylurea; Liver disease; Liver enzyme

\section{Introduction}

\section{Idiosyncratic Reactions}

For some drugs, a genetic or acquired abnormality must exist in a particular metabolic pathway for a toxic reaction to take place in other cases; the reactions are typically associated with a drug concentration and often respond to simply lowering the dose of the drug.
Idiosyncratic reactions tend to occur without association to particular blood concentrations or specifically identified metabolic abnormalities. For example, sulfonylureas like glipizide and antibiotics like ciprofloxacin have caused severe liver disease, resulting in the need for transplantation in a very small group of patients [1,2]. Idiosyncratic reactions are rare and are sometimes described as liver hypersensitivity to a drug. 


\section{Advances in Pharmacology and Clinical Trials}

\section{Allergic Hepatitis}

Allergic reactions in the liver can be caused by many drugs and result in many different kinds of hepatic damage. Trimethoprim sulfamethoxazole and penicillinase-resistant penicillins such as dicloxacillin induce a reaction typical of hepatic hypersensitivity in a few patients. The reaction usually develops within 4 weeks of the start of therapy [3,4]. It is marked by fever, pruritus, rash, eosinophilia, arthritis, and hemolytic anemia. The formation of granulomas within the liver is often seen on biopsy. The reaction reverses with discontinued therapy and reappears upon rechallenge. Most antibiotics have been associated with this type of reaction, including the fluoroquinolones, macrolides, and $\beta$-lactams. Allopurinol also has been associated with a number of reports of hypersensitivity reactions involving the liver. The onset of symptoms is 1 to 6 weeks after initiation of therapy. The incidence, like all the allergic liver reactions, is low, estimated at less than $1 \%$. The clinical presentation includes eosinophilia, fever, rash, and arthritis, as previously mentioned. The biopsy may show a pattern of fibrin-ring granulomas similar to those seen in $\mathrm{Q}$ fever [5].

\section{Toxic Hepatitis}

Toxic reactions are predictable, often dose-related effects in the liver due to specific agents. When taken in overdose, acetaminophen becomes bio-activated to a toxic intermediate known as $N$-acetyl- $p$-benzoquinone imine (NAPQI). NAPQI is very reactive, with a high affinity for sulfhydryl groups. The amino acid glutathione provides a ready source of available sulfhydryl groups within the hepatocyte. When the liver's glutathione stores are depleted and there are no longer sulfhydryl groups available to detoxify this metabolite, it begins to react directly with the hepatocyte. Replenishing the liver's sulfhydryl capacity through the administration of $\mathrm{N}$ acetyl-cysteine early after ingestion of the overdose halts this process. Acetaminophen's toxicity occurs in four stages. During the first hours after ingestion, some patients report mild symptoms of nausea and vomiting, but no elevations of the commonly measured liver enzymes are seen but for 40 to 50 hours after ingestion do elevations in the liver enzymes begin. Reye's syndrome is an aggressive form of toxic hepatitis often associated with aspirin use in children. Valproate toxicity can also present in this pattern. Early in the process of Reye's syndrome, mitochondrial dysfunction leads to the depletion of acyl coenzyme A and carnitine. Fatty acids accumulate and gluconeogenesis is impaired, resulting in hypoglycemia. A concurrent disruption of the urea cycle occurs, leading to a decrease in the removal of ammonia and a slowing of protein use. A threefold rise in the blood ammonia level and an increase in the prothrombin time are common findings. In advanced stages of Reye's syndrome, many patients develop intracranial hypertension that can be life threatening and refractory to therapy $[6,7]$.

\section{Chronic Active Toxic Hepatitis}

Dantrolene, isoniazid, phenytoin, nitrofurantoin, and trazodone have been reported in association with a type of autoimmune mediated disease in the liver. Patients experience periods of symptomatic hepatitis followed by periods of convalescence, only to repeat the experience months later. It is a progressive disease with a high mortality rate and is more common in females than males. Antinuclear antibodies appear in most patients. These drugs appear to form anti organelle antibodies [8]. The exact identification of a causative agent is sometimes difficult since diagnosis requires multiple episodes occurring long after exposure to the offending drug.

\section{Toxic Cirrhosis}

The scarring effect of hepatitis in the liver leads to the development of cirrhosis. Some drugs tend to cause such a mild case of hepatitis that it may not be detected. Mild hepatitis can be easily mistaken for a more routine generalized viral infection. If the offending drug or agent is not discontinued, this damage will continue to progress. The patient eventually presents not with hepatitis, but with cirrhosis. Methotrexate causes periportal fibrosis in most patients who experience hepatotoxicity. The lesion results from the action of a bio-activated metabolite produced by cytochrome P450. This process has most commonly been noted in patients treated for psoriasis and arthritis. The extent of damage can be reduced or controlled by increasing the dosage interval to once weekly or by routine use of folic acid supplements. Vitamin A is normally stored in liver cells, and causes significant hypertrophy and fibrosis when taken for long periods in high doses. Hepatomegaly is a common finding, along with ascites and portal hypertension. In patients with vitamin A toxicity, gingivitis and dry skin are also very common. This is accelerated by ethanol, which competes with retinol for aldehyde dehydrogenase [9].

\section{Mechanisms of Drug-Induced Liver Disease}

\section{Centrolobular Necrosis}

Centro-lobular necrosis is often a dose-related, predictable reaction secondary to drugs such as acetaminophen; however, it also can be associated with idiosyncratic reactions, such as those caused by 


\section{Advances in Pharmacology and Clinical Trials}

halothane. Also called direct or metabolite-related hepatotoxicity, centrolobular necrosis is usually the result of the production of a toxic metabolite. The damage spreads outward from the middle of a lobe of the liver. Patients suffering from Centro-lobular necrosis tend to present in one of two ways, depending on the extent of necrosis. Mild drug reactions, involving only small amounts of parenchymal tissue, may be detected as asymptomatic elevations in the serum transaminases. If the reaction is diagnosed at this stage, most of these patients will recover with minimal cirrhosis and thus minimal chronic liver impairment. More severe forms of centro-lobular necrosis are accompanied by nausea, vomiting, upper abdominal pain, and jaundice [10].

\section{Steatohepatitis}

Steato-hepatitis (also known as steato-necrosis) is a specialized type of acute necrosis resulting from the accumulation of fatty acids in the hepatocyte. Drugs or their metabolites that cause steato-necrosis do so by affecting fatty-acid oxidation within the mitochondria of the hepatocyte. Hepatic vesicles become engorged with fatty acids, eventually disrupting the homeostasis of the hepatocyte. The liver biopsy is marked by a massive infiltration by polymorphonuclear leukocytes, degeneration of the hepatocytes, and the presence of Mallory bodies. Alcohol is the drug that most commonly produces steato-necrotic changes in the liver. When alcohol is converted into acetaldehyde, the synthesis of fatty acids is increased. When the hepatocyte has become completely engorged with microvesicular fat, it often breaks open, spilling into the blood. If enough hepatocytes break open, an inflammatory response begins. If the offending agent is withdrawn before significant numbers of hepatocytes become necrotic, the process is completely reversible without long-term sequelae. In nonalcoholic steato-hepatitis the same endpoint is often achieved through oxidation of lipid peroxidases. Tetracycline produces steato-hepatitis and steatosis. The lesions are characterized by large vesicles of fat found diffused throughout the liver. The development of this reaction is related to the high concentrations achieved when tetracycline is given intravenously and in doses greater than $1.5 \mathrm{~g} /$ day. The mortality of tetracycline steatohepatitis is very high $(70 \%$ to $80 \%)$, and those who do survive often develop cirrhosis. Sodium valproate also can produce steato-necrosis through the process of bioactivation. Cytochrome P450 converts valproate to D4-valproic acid, a potent inducer of microvesicular fat accumulation. Patients experiencing steato-hepatitis may present with abdominal fullness or pain as their only complaint. Patients with more severe steato-necrosis will present with all the symptoms characteristic of alcoholic hepatitis such as nausea, vomiting, steatorrhea, abdominal pain, pruritus, and fatigue [11].

\section{Phospholipidosis}

Phospholipidosis is the accumulation of phospholipids instead of fatty acids. The phospholipids usually engorge the lysosomal bodies of the hepatocyte. Amiodarone has been associated with this reaction. Patients treated with amiodarone who develop overt hepatic disease tend to have received higher doses of the drug. These patients also have higher amiodarone to $\mathrm{N}$-desethyl-amiodarone ratios, indicating a greater accumulation of the parent compound. Amiodarone and its major metabolite $\mathrm{N}$ desethyl-amiodarone remain in the liver of all patients for several months after therapy is stopped. Usually the phospholipidosis develops in patients treated for more than 1 year. The patient can present with either elevated transaminases or hepatomegaly, jaundice is rare [12].

\section{Generalized Hepatocellular Necrosis}

Generalized hepatocellular necrosis mimics the changes associated with the more common viral hepatitis. The onset of symptoms is usually delayed as much as a week or more after exposure to toxin. Bio-activation is often important for toxic hepatitis to develop, but may not be the immediate cause of damage. Many drugs that are associated with toxic hepatitis produce metabolites that are not inherently toxic to the liver. Instead, they act as haptens, binding to specific cell proteins and inducing an autoimmune reaction. The rate of bioactivation can vary between males and females and between individuals of the same sex. The cytochrome P450 system (CYP) tends to metabolize lipophilic substrates which are actively pumped into the hepatocyte by an organic anion (or cation) transporting protein. The CYP subspecies $1 \mathrm{~A}, 2 \mathrm{~B}$, $3 \mathrm{~A}$, and $4 \mathrm{~A}$ are regulated by the highly inducible xenobiotic receptor on complementary DNA. The receptor is found in the liver, and to a lesser extent in the cells lining the intestinal tract, and is responsible for cholesterol catabolism and bile acid homeostasis. The activity of this receptor is subject to genetic polymorphism as well. This results in a wide variation in the sensitivity of the population to generalized hepatocellular necrosis and other forms of hepatic damage. The long-term administration of isoniazid can lead to hepatic dysfunction in $10 \%$ to $20 \%$ of those receiving the drug. Yet severetoxic hepatitis develops in only $1 \%$ or less of this population. The $\mathrm{N}$ acetyltransferase 2 (NAT2) genotype appears to play a role in determining a patient's relative risk. In one study, patients with the slowtype NAT2 genotype had a 28-fold greater risk of developing serum aminotransferase 


\section{Advances in Pharmacology and Clinical Trials}

elevations than did patients with the fast-type NAT2 genotype. Isoniazid is metabolized by several pathways, acetylation being the major pathway. It is acetylated to acetyl isoniazid, which, in turn, is hydrolyzed to acetylhydrazine [13]. The acetylhydrazine, and to a lesser extent the acetyl isoniazid, are directly toxic to the cellular proteins in the hepatocyte, but rapid acetylators also detoxify acetyl hydrazine very rapidly, converting it to diacetyl hydrazine (a nontoxic metabolite). Ketoconazole produces generalized hepatocellular necrosis or milder forms of hepatic dysfunction in $1 \%$ to $2 \%$ of patients treated for fungal infections. This reaction is fatal in high numbers of patients infected with the human immunodeficiency virus. The onset is usually early in therapy, although it can be delayed until several months into therapy. In immune-compromised patients in whom ketoconazole is used for long periods of time, special care should be taken to watch for changes in liver function [14].

\section{Cholestatic Jaundice}

Cholestatic jaundice, or cholestasis, can be classified by the area of the bile canalicular or ductal system that is impaired. Canalicular cholestasis is very often associated with long-term high-dose estrogen therapy. Clinically, these patients are often asymptomatic and present with mild to moderate elevations of serum bilirubin [15]. An intravenous form of vitamin E, $\alpha$-tocopherol acetate, causes cholestatic jaundice primarily involving the canalicular duct in premature infants. The incidence of this reaction in those receiving this formulation was high $(>10 \%)$ and the mortality even higher $(>50 \%)$. Hepatocellular cholestasis is a much more serious form of cholestatic jaundice that involves both the parenchyma and bile canalicular cells. The administration of total parenteral nutrition for periods greater than 1 week induces cholestatic changes and nonspecific enzyme elevations in some patients. Patients with low serum albumin concentrations may be at greater risk than patients with normal serum albumin concentrations. This reaction also has been reported to occur rarely with sulfonamides, sulfonylureas, erythromycin estolate and ethylsuccinate, captopril, lisinopril, and other phenothiazines. The Table 1 revealed the environmental hepatotoxin which causes liver related problems and Table 2 revealed pattern of hepatic-enzyme versus hepatic-lesion.

\begin{tabular}{|c|c|}
\hline Hepatotoxin & Associated Occupations at Risk for Exposure \\
\hline Arsenic & Chemical plant, construction, agricultural workers \\
\hline Carbon tetrachloride & Chemical plant workers, laboratory technicians \\
\hline Copper & Plumbers, outdoor sculpture artists, copper foundry workers \\
\hline Dimethylforamide & Chemical plant workers, laboratory technicians \\
\hline 2,4-Dichlorophenoxyacetic acid & Chemical plant workers, laboratory technicians \\
\hline Fluorine & Chemical plant, agricultural workers, laboratory technicians \\
\hline Toluene & Printers, dye workers, cleaners, laboratory technicians \\
\hline Trichloroethylene & Plastics plant workers, also found as a river pollutant \\
\hline Vinyl chloride & .
\end{tabular}

Table 1: Environmental Hepatotoxins and Associated Occupations at Risk for Exposure.

\begin{tabular}{|c|c|c|c|}
\hline Enzyme & Necrotic & Cholestatic & Chronic \\
\hline Alkaline phosphatase & $<100 \%$ of normal & $>200 \%$ of normal & $<100 \%$ of normal \\
\hline 5'-Nucleotidase & $<100 \%$ of normal & $>200 \%$ of normal & $<100 \%$ of normal \\
\hline$\gamma$ Glutamyltransferase & $<100 \%$ of normal & $>200 \%$ of normal & $>100 \%$ of normal \\
\hline Aspartate aminotransferase & $>200 \%$ of normal & $<100 \%$ of normal & $>100 \%$ of normal \\
\hline Alanine aminotransferase & $>200 \%$ of normal & $<100 \%$ of normal & $>100 \%$ of normal \\
\hline Lactate dehydrogenase & $>200 \%$ of normal & $<100 \%$ of normal & $<100 \%$ of normal \\
\hline
\end{tabular}

Table 2: Relative Patterns of Hepatic Enzyme Elevation versus Type of Hepatic Lesion.

\section{Measurement of Liver Function}

A good compound for a liver function test would theoretically be (a) nontoxic and lacking any pharmacologic effect (b) either rapidly and completely absorbed orally or easily administered via a peripheral vein (c) eliminated only by the liver; and (d) easily measured (drug and its metabolite) in blood, saliva, or urine [16]. Several tests are used in research settings and in liver transplant patients to indicate liver function. Tests 


\section{Advances in Pharmacology and Clinical Trials}

such as sulfobromophthalein, indocyanine green or sorbitol measure qualities of hepatic clearance. There are also a few drugs that have been used to test liver function. Sorbitol's advantage over indocyanine green is a much lower incidence of allergic reactions. It is partially cleared by the kidney, and urine levels must also be determined during the test [17]. A good estimate of hepatic clearance can be obtained by serial blood levels of a variety of hepatically eliminated drugs if an assay is locally available. Ultrasound and CT imaging can be used on a periodic basis to monitor for the development of fibrosis or vascular lesions in the liver and for hepatocellular carcinomas [18]. If a liver biopsy has been performed, the injury should be classified by the histologic findings. In cases in which there is no biopsy, the pattern of liver enzyme elevation can estimate the type of injury. Hepatocellular injuries are marked by elevations in transaminase that are at least two times normal. If the alkaline phosphatase is also elevated, then a hepatocellular lesion is still suspected when the elevation of ALT is notably higher than the elevation of alkaline phosphatase. If the magnitude of elevation is nearly equal between ALT and alkaline phosphatase, then the lesion is likely cholestatic. A liver injury is acute if it lasts less than 3 months, and it is considered chronic after 3 months of consistent symptoms or enzyme elevation. A liver injury is severe if the patient has marked jaundice, if the prothrombin time does not improve by more than $50 \%$ after the administration of vitamin $\mathrm{K}$, or if encephalopathy is detectable. If an acute liver injury progresses from normal to severe in a matter of a few days or weeks, it is considered fulminant [19].

\section{Monitoring}

The serum transaminases AST and ALT are the most commonly used transaminases in the clinical setting. There are often no set rules available for a particular drug.

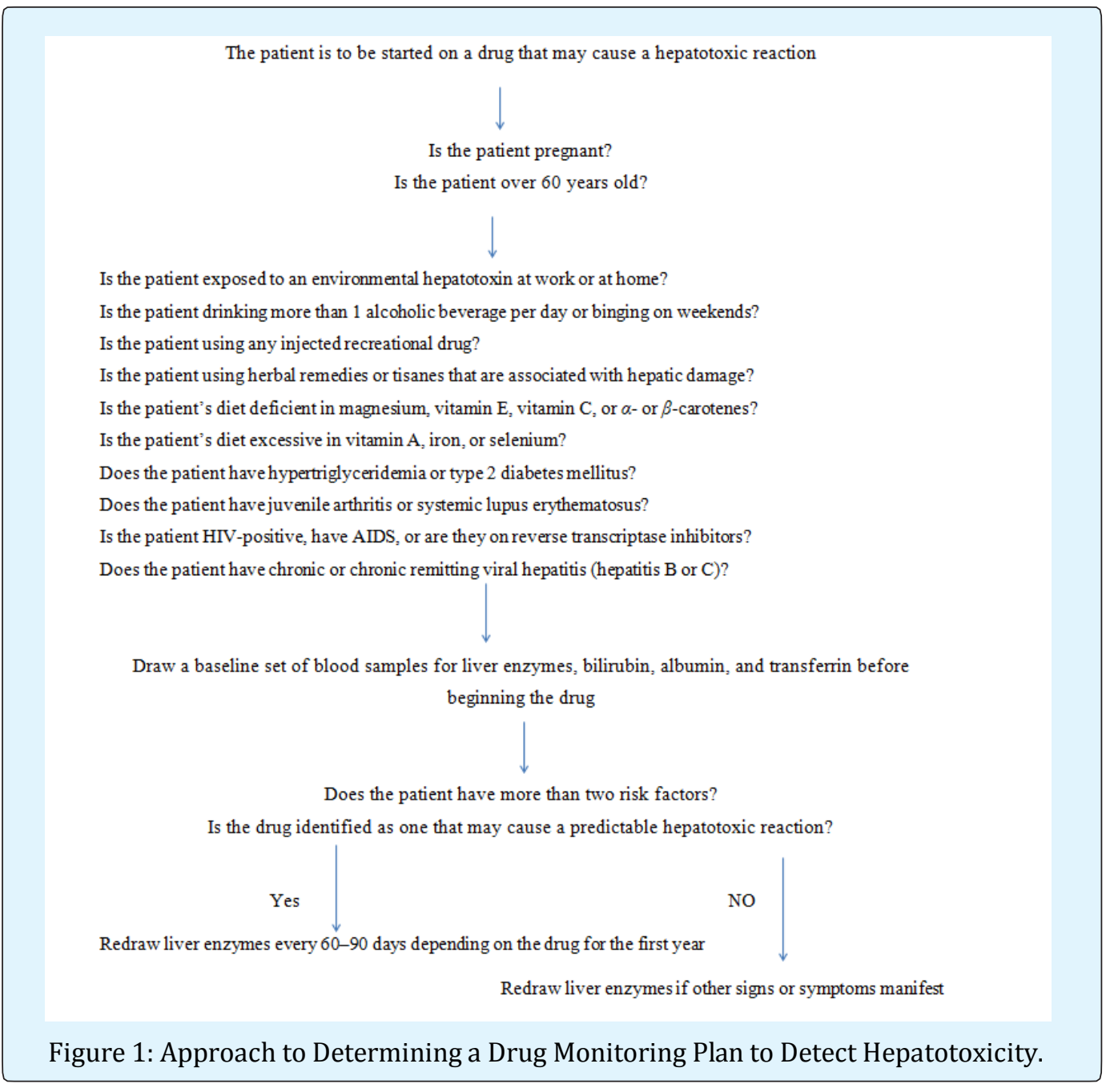




\section{Advances in Pharmacology and Clinical Trials}

The general guidelines found in flow chart, which is to be followed and it can help in determining a monitoring schedule for drugs where no prior recommendations are published. Concentrations of these enzymes should be obtained about every 4 weeks, depending on the reported characteristics of the reaction in question. Methotrexate should be monitored every 4 weeks, because toxicity usually develops over a period of several weeks to months [20]. In addition, some recommend that sulfobromophthalein or indocyanine-green excretion studies be performed on a regular basis and that patients treated for very long periods of time should have a liver biopsy performed every 12 months [21].

If no toxicity is manifested during the first year of therapy, then redraw liver enzymes every 6-12 months; assess liver for cirrhosis every 1-2 years by ultrasound and every 4-6 years by CT or MRI scan; biopsy as directed by other findings.

\section{Conclusion}

There is a very little data on specific increased or decreased susceptibilities to drug induced liver injury with increasing age, but a few observations are important. As the normal liver age hepatic blood flow decreases as much as $30 \%$ by age 65 . This decrease in liver blood flow increases the bioavailability of highly extracted drugs, such as labetolol, propranolol, lidocaine, and chlormethiazole. The risk of hepatotoxicity is apparently greater in person 70 years old or older, according to data from the United Kingdom. There $>5 \%$ of all adverse reactions in persons 70 years old or older occur in the liver, while only $2 \%$ of all adverse reactions in children (0-14 years) occur in the liver. The potential mortality from hepatotoxic reaction is also higher in the elderly, who often have concurrent decreases in renal or cardiac function. Neoplastic disease and hepatocellular carcinomas are also more common in the elderly than in younger patients. A liver injury is acute if it lasts less than 3 months, and it is considered chronic after 3 months of consistent symptoms or enzyme elevation. A liver injury is severe if the patient has marked jaundice, if the prothrombin time does not improve by more than $50 \%$ after the administration of vitamin $\mathrm{K}$, or if encephalopathy is detectable. If an acute liver injury progresses from normal to severe in a matter of a few days or weeks, it is considered fulminant.

\section{References}

1. Dourakis SP, Tzemanakis E, Sinani C, Georgie K, Hadziyannis SJ, et al. (2000) Gliclazide-induced acute hepatitis. The European Journal of Gastroenterology \& Hepatology 12: 119-121.

2. Villeneuve JP, Davies C, Cote JJ (1995) Suspected ciprofloxacin-induced hepatotoxicity. The Annals of Pharmacotherapy 29: 257-259.

3. Olsson R, Wiholm BE, Sand C, Zettergren L, Hultcrantz $\mathrm{R}$, et al. (1992) Liver damage from flucloxacillin, cloxacillin and dicloxacillin. Journal of Hepatology 15(1-2): 154-161.

4. Lindgren A, Olsson R (1994) Liver reactions from trimethoprim. Journal of Internal Medicine 236(3): 281-284.

5. Vanderstigel M, Zafrani ES, Deyone JL, Schaeffer A, Portos JL, et al. (1986) Allopurinol hypersensitivity syndrome as a cause of hepatic fibrin granulomas. Gastroenterology 90(1): 188-190.

6. Belay ED, Bresee JS, Holman RC, Khan AS, Shahriari A, et al (1999) Reye's syndrome in the United States from 1981 through 1997. New England Journal of Medicine 340(18): 1377-1382.

7. Monto AS (1999) The disappearance of Reye's syndrome - a public health triumph. New England Journal of Medicine 340(18): 1423-1424.

8. Beane PH, Bourdi M (1993) Autoantibodies against cytochrome $\mathrm{P} 450$ in druginduced autoimmune hepatitis. The Annals of the New York Academy of Sciences 685: 641-645.

9. Leo MA, Lieber CSJ (1999) Alcohol, vitamin A, and beta-carotene: adverse interactions, including hepatotoxicity and carcinogenicity. The American Journal of Clinical Nutrition 69(6): 1071-1085.

10. Fontana RJ, McCashland TM, Benner KG, Appelman HD, Gunartanam NT, et al. (1999) Acute liver failure associated with prolonged use of bromfenac leading to liver transplantation. The Acute Liver Failure Study Group. Liver Transplant Surgery 5: 480-484.

11. Lullman H, Lullman R, Wasserman O (1975) Druginduced phospholipoidosis II. Tissue distribution of the amphiphilic drug chlorphentermine. CRC critical reviews in toxicology 4(2): 185-218.

12. Chang CC, Petrelli M, Tomashefski JF, McCullough AJ (1999) Severe intrahepatic cholestasis caused by amiodarone toxicity after withdrawal of the drug: A 


\section{Advances in Pharmacology and Clinical Trials}

case report and review of the literature. Archives of pathology \& laboratory medicine 123(3): 251-256.

13. Kergueris MF, Bourin M, Larousse C (1986) Pharmacokinetics of isoniazid. Influence of age. European Journal of Clinical Pharmacology 30(3): 335-340.

14. Van Puijenbroek EP, Metselaar HJ, Berghuis $\mathrm{PH}$, Zondervan PE, Stricker BH, et al. (1998) Acute hepatocytic necrosis during ketoconazole therapy for treatment of onychomycosis. National Foundation for Registry and Evaluation of Adverse Effects. Nederlands Tijdschrift voor Geneeskunde 142(44): 2416-2418.

15. Foitl DR, Hyman G, Leftowitch JH (1989) Jaundice and intrahepatic cholestasis following high-dose megesterol acetate for breast cancer. Cancer 63(3): 438-439.

16. Barstow L, Smith RE (1990) Liver function assessment by drug metabolism. Pharmacotherapy 10(4): 280-288.

17. Zech J, Lange H, Bosch J, Jaime Bosch, Susanne Pohl, et al. (1988) Steady-state extrarenal sorbitol clearance as a measure of hepatic plasma flow. Gastroenterology 95: 749-759.

18. Mathieu D, Kobeiter H, Maison P, Rahmouni A, Cherqui D, et al (2000) Oral contraceptive use and focal nodular hyperplasia of the liver. Gastroenterology 118(3): 560-564.

19. (1990) Anonymous. Standardization of definitions and criteria of causality assessment of adverse drug reactions, drug-induced liver disorders. Report of an international consensus meeting. International journal of clinical pharmacology, therapy and toxicology 28: 317-322.

20. Newman M, Auerbach R, Feiner H, Holzman RS, Shupack J, et al. (1989) the role of liver biopsies in psoriatic patients receiving long-term methotrexate treatment: Improvement in liver abnormalities after cessation of treatment. Archives of dermatology 125(9): 1218-1224.

21. O'Connor GT, Olmstead EM, Zug K, Baughman RD, Beck JR, et al. (1989) Detection of hepatotoxicity associated with methotrexate therapy for psoriasis. Archives of Dermatology 125(9): 1209-1217.

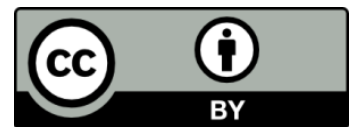

